\title{
Analysis of Guidance Laws with Non-monotonic Line-of-Sight Rate Convergence
}

\author{
Seokwon Lee, Namhoon Cho, Member, IEEE, Hyo-Sang Shin, Member, IEEE
}

\begin{abstract}
This study presents analyses of guidance laws that involve non-monotonic convergence in heading error from a new perspective based on an advanced stability concept. Pure proportional navigation with range-varying navigation gain is considered, and the gain condition to guarantee asymptotic convergence to the collision course is investigated while allowing the heading error to exhibit patterns that involve intermediate diversion. The extended stability criterion considered in this study allows local increase of the function in some finite intervals, which is less conservative than the standard stability theorem. The existing guidance laws involving intentional modulation of the heading error as well as the design of the navigation gain are discussed with respect to the new stability criterion.
\end{abstract}

Index Terms-Stability Theory, Missile Guidance, Proportional Navigation Guidance, Trajectory Modulation, Observability Enhancement, Weaving Manoeuvre

\section{INTRODUCTION}

Advanced homing guidance strategies that are developed to enhance the capabilities of missiles, such as target observability and survivability, usually exploit manoeuvre patterns with alternating line-of-sight rates. The primary objective of homing guidance is to enter a collision course, whereas the other manoeuvre goals benefit from larger deviation in the flight trajectory from the collision course, inevitably yielding non-monotonic behaviours in the heading error. Dual-control guidance strategies such as adaptive intermittent manoeuvre (AIM) strategy [1]-[3] and optimal guidances [4]-[6] intentionally involve the line-of-sight (LOS) rate excitation to suffice an observability condition [2], [7]-[12] while sacrificing the convergence to the collision course. Also, evasive guidance strategies were designed for anti-ship missiles to accomplish target interception while enhancing the survivability against ship defence systems [13]-[15], where the weaving motion leads to deviation from the collision course. Such oscillatory or rapidly-changing manoeuvres cause an abrupt and drastic change of flight path. Therefore, in order to prevent possible instability arising from the abrupt behaviours, stability analysis is a prerequisite in the design process.

The standard stability theory is limited in analysing the stability of the guidance laws requiring non-monotonic LOS rate convergence due to its conservative nature. In general, the candidate function that represents convergence to a collision course is expressed by the LOS rate or heading error. Under the standard stability theory, the asymptotic stability of the

S. Lee, N. Cho, and H.-S. Shin are with the School of Aerospace, Transport and Manufacturing, Cranfield University, MK43 OAL Cranfield, U.K., E-mail: (seokwon.lee@cranfield.ac.uk); (n.cho@cranfield.ac.uk); (h.shin@cranfield.ac.uk) guidance loop is analysed by showing that the derivative of the function is negative definite [?], [?], [?], [16]-[20]. However, negative definiteness of the derivative limits the asymptotic stability of the system to the monotonic convergence of the LOS rate. For this reason, while the standard stability theory can explain the monotonically convergent response of the generalised energy-optimal guidance law [21]-[25], it is inadequate for describing the asymptotic stability of guidance laws, including non-monotonic motions. Therefore, an extended stability theory that provides a relaxed stability criterion than the standard stability theory is required to analyse the nonmonotonic characteristics for these types of guidance laws.

Asymptotic stability of time-varying nonlinear systems has been widely investigated in many ways to relax and extend the standard stability that requires negative definiteness of the time derivative of the function for all time instances to guarantee asymptotic stability. Krasovskii-LaSalle theorem requires the time derivative to be negative semi-definite in proving asymptotic stability whenever the weak zero-state detectability property holds [26]. The averaging methods have been developed to determine the exponential stability of the original system through analysis on the exponential stability of the time-invariant averaged system, assuming that the timevarying part evolves sufficiently fast [27]. On the other hand, the theorem expressed in terms of solutions at sampling instances was introduced in [28]. The notion of uniform asymptotic stability of time-varying nonlinear systems was addressed by using a function whose value decreases along with the solutions at sampling instances. Notably, this result implies that the asymptotic convergence does not necessarily require the time-derivative of the function to be negative definite for all time instances. Further studies based on [28] have been carried out in regard to the exponential stability [29], showing that the global asymptotic stability of the averaged system implies uniform semi-global practical stability of the actual system.

The main objective is to investigate a less conservative condition of the guidance loop stability than the existing stability criterion and thus provide flexibility in designing guidance laws. Motivated by the results in Ref. [28], this study aims to provide a new perspective based on the extended stability theory that particularly suits the analysis of the proportional navigation guidance (PNG) variants with non-monotonic convergence to the collision course due to trajectory modulation. The analysis focuses on the PNG with a range-varying navigation gain as it represents a wealth of guidance strategies. Since the asymptotic stability is defined with respect to the solution behaviour as time tends to infinity 
while an interception problem ends at a finite time, a new transformation of variable is introduced to change the associated equations into the form suitable for stability analysis. A sufficient condition for the asymptotic convergence of the heading error to zero, i.e., convergence to the collision course, in the transformed domain is established as a new criterion for the guaranteed closed-loop stability. Subsequently, a condition guaranteeing the satisfaction of the corresponding stability criterion is derived in terms of the navigation gain function. The stability of existing guidance laws is investigated in view of the proposed criterion. Also, new guidance laws that entail oscillatory responses in the heading error for trajectory shaping while ensuring asymptotically stable convergence are proposed based on the extended stability theory.

The analysis presented in this study has several implications. First, the extended stability criterion discussed in this study is less conservative than the classical counterpart that demands strict negative definiteness of the rate of the function and results in monotonic convergence of LOS rate to zero in the entire time interval. Hence, the extended stability criterion allows us to interpret the non-monotonic response characteristics of the advanced guidance strategies. Second, it enables stabilityensured design for a wider class of PNG variants, particularly the ones in which line-of-sight rate may grow in intermediate time intervals before the end of the engagement. In this way, this study extends our understanding of advanced trajectorymodulated guidance laws in a control-theoretic context.

The remainder of this study is organised as follows. Mathematical foundations and equations of motion are described in Sec. II. In Sec. III, the extended stability criterion is presented, and existing guidance laws are investigated from the renewed viewpoint based on stability. Design of new guidance laws based on the stability condition is demonstrated as well. Simulation examples are provided to verify the theoretical findings in Sec. IV. Summary and concluding remarks are presented in Sec. V.

\section{Preliminaries}

This section describes mathematical foundations for this study. This study begins with the stability theory in Sec. II-A. The essential theorem addressed in Ref. [28] is reproduced in a simpler form for completeness. The closed-loop missile system governed by PNG are described in Sec.II.B. Since the considered system is described in terms of the range, a proper treatment is needed to address the closed-loop stability in the considered stability sense. Sec. II-C describes how the governing equations is transformed for the stability analysis.

\section{A. Lyapunov Stability Theory for Continuous-Time Nonlinear Systems}

This section briefly reviews the stability of the dynamical system. The non-autonomous nonlinear dynamics represented in time can be summarised as follows.

$$
\dot{\mathbf{x}}=\mathbf{f}(\mathbf{x}(t), t)
$$

where $\mathbf{f}(\mathbf{x}, t)$ is piecewise continuous in $t \in \mathbb{R}$ and Lipschitz continuous in $\mathbf{x} \in U$, and the origin is an equilibrium point satisfying $\mathbf{f}(\mathbf{x}, t) \rightarrow \mathbf{0}, \forall \mathbf{x} \rightarrow 0, \forall t \in \mathbb{R}$. The definition of the (asymptotic) stability and a relaxed asymptotic stability condition are given by

Definition 1. ( [27]) Let $\phi(t, \mathbf{x}(0))$ be the solution of Eq. (1) starting from $\mathbf{x}(0)$. Then,

- (Stable): if $\forall \epsilon>0, \exists \delta\left(\epsilon, t_{0}\right)>0$ such that $\|\mathbf{x}(0)\| \leq$ $\delta \Rightarrow\|\mathbf{x}(t)\| \leq \epsilon, \forall t \geq t_{0}$. If $\delta=\delta(\epsilon)>0$ is independent of $t_{0}$, it is uniformly stable.

- (Attractive): If $\|\mathbf{x}(0)\| \leq \delta \Rightarrow \lim _{t \rightarrow \infty} \phi(t, \mathbf{x}(0)) \rightarrow 0$

- (Asymptotically stable) If the origin is stable as well as attractive.

Theorem 1. ( [28]) Consider a system (1) with $\mathbf{f}$ being locally Lipschitz continuous, and function $V: U \times \mathbb{R}^{+} \rightarrow \mathbb{R}^{+}$with $U \subset W$ an open neighbourhood of the origin. Then the origin is asymptotically stable if

- (C1) $V(\mathbf{x}, t)$ is decrescent and positive definite, i.e., $\alpha_{1}(\|\mathbf{x}\|) \leq V(\mathbf{x}, t) \leq \alpha_{2}(\|\mathbf{x}\|)$ where $\alpha_{1}(\cdot), \alpha_{2}(\cdot): \mathbb{R}^{+} \rightarrow$ $\mathbb{R}$ belong to class $\mathcal{K}$ function.

- (C2') For all $t$ and $\mathbf{x}(t)$, there exists a finite $T>0$ and a class $\mathcal{K}$ function $\gamma(\cdot)$ satisfying

$$
V(\mathbf{x}(t+T), t+T)-V(\mathbf{x}(t), t) \leq-\gamma(\|\mathbf{x}\|)<0
$$

A remarkable property of Theorem 1 is that the negative definiteness of $\dot{V}(\mathbf{x}, t)$ is no longer needed. The standard Lyapunov stability condition [27] requires $\dot{V}$ to be negative definite to ensure the asymptotic stability. However, the standard stability criterion poses a limitation in that it demands the Lyapunov function to decrease monotonically along with the solution. In contrast, the decrement condition over a finite interval [28] allows energy-growing and exploratory response in achieving the asymptotic stability. Hence, the extended theory enables stability-oriented design and analysis of control systems that exhibit more general behavioural patterns in their solutions.

\section{B. Engagement Kinematics and Proportional Navigation Guidance}

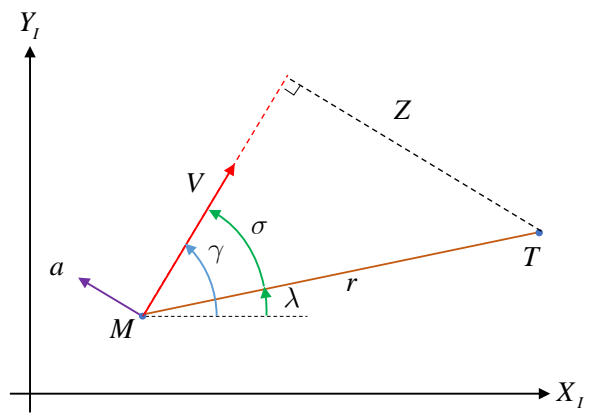

Fig. 1: Planar Engagement Guidance Geometry

This section describes the kinematic equations of motion associated with the trajectory guided by PNG. Consider the ideal planar engagement against stationary target. Figure 1 presents the guidance geometry and the definition of variables. 
In Fig. $1, r$ and $\lambda$ represent the range and the line-of-sight angle, respectively. $\gamma$ is the flight path angle, and $\sigma=\gamma-\lambda$ is the lead angle. $V_{M}$ and $a_{M}$ denote the speed and the lateral acceleration, respectively. The engagement kinematics is given by

$$
\begin{aligned}
\dot{r} & =-V_{M} \cos \sigma \\
\dot{\lambda} & =-\frac{V_{M}}{r} \sin \sigma \\
\dot{\sigma} & =\frac{V_{M}}{r} \sin \sigma+\frac{a_{M}}{V_{M}}
\end{aligned}
$$

In the equations of motion, the acceleration $a_{M}$ is regarded as the guidance command to steer the missile to intercept the target. Using Eqs. (2b) and (2c), the relative motion can be expressed in terms of $a_{M}$ as

$$
r \ddot{\lambda}+2 \dot{r} \dot{\lambda}=-a_{M} \cos \sigma
$$

Pure PNG (PPNG) generates the acceleration command perpendicular to the velocity to be proportional to the LOS rate as

$$
a_{M}=N(r) V_{M} \dot{\lambda}
$$

where $N(r)$ is the navigation gain. By substituting the PPNG command into Eq. (3), the closed-loop differential equation can be written as

$$
r \ddot{\lambda}+(2-N(r)) \dot{r} \dot{\lambda}=0
$$

Note that the origin $(r, \dot{\lambda}, \sigma)=0$ is not an equilibrium because $\dot{r} \neq 0$ as $\sigma=0$. Hence, one cannot draw a conclusion with regard to the guarantee of interception through analysing the stability of an equilibrium point when treating the range as a state variable. Instead, nullification of the heading error determines stable entrance into a collision course with the target. The following condition is introduced for the positive closing speed.

Assumption 1. The range $r$ is monotonically decreasing throughout the engagement, i.e., $\dot{r}<0$ for all $t \geq t_{0}$.

Assumption 1 holds as long as $|\sigma|<\pi / 2$. Assumption 1 ccan be justified in many guidance schemes formulated with range as the independent variable, and in the methods developed to respect the seeker's field-of-view limit during the engagement [30]-[34]. The one-to-one correspondence between time and range allows us to represent Eq. (2) in the range domain. Considering the transformation $\frac{d}{d t}=\dot{r} \frac{d}{d r}$, Eq. (5) can be expressed as

$$
\frac{d \dot{\lambda}}{d r} \triangleq \frac{K(r)}{r} \dot{\lambda}=\frac{N(r)-2}{r} \dot{\lambda}
$$

Using this treatment, the zero heading error equilibrium can be determined as stable if $\dot{\lambda}$ in Eq. (6) converges to zero as $r \rightarrow$ 0 . It is worth noting that the differential equation is evolved from $r=r_{0}$ to $r=r_{f}$, which is the opposite to the time domain evolution. Also, the feedback gains in Eqs. (6) is denominated in $r$, which makes violates the local Lipschitz continuity of $f=\frac{K(r)}{r} \dot{\lambda}$ near $r=0$ required for the Lyapunov stability theorems stated in Theorem 1.

\section{Reformulation of Kinematics}

Let us introduce a new variable $\tau=-\ln r$. Then, the oneto-one correspondence between $\tau$ and $r$ permits change of the independent variable. Equation (6) can be expressed in terms of $\tau$ as

$$
\frac{d \dot{\lambda}}{d \tau}=-K(\tau) \dot{\lambda}
$$

Note that the state equation, Eq. (7), is a linear rangevarying (LRV) system, which makes it easier to analyse the performance of guidance laws. If the solution of the closedloop system exists and is unique, the right hand side of the equation should be integrable. For further developments, let the gain $K(r)$ be bounded from below and above as $\underline{K} \leq K(r) \leq \bar{K} \in \mathbb{R}^{+}$. The boundedness condition of $K$ is sufficient for Lipschitz condition, which is required for the uniqueness of the solution and stability analysis. That is,

$$
\left|\frac{d \dot{\lambda}\left(\tau_{1}\right)}{d \tau}-\frac{d \dot{\lambda}\left(\tau_{2}\right)}{d \tau}\right|=\left|K(\tau) \dot{\lambda}_{1}-K(\tau) \dot{\lambda}_{2}\right| \leq \bar{K}\left|\dot{\lambda}_{1}-\dot{\lambda}_{2}\right|
$$

The trajectory of $\dot{\lambda}$ can be expressed as the fundamental function $\Phi\left(\tau, \tau_{0}\right)$.

$$
\dot{\lambda}(\tau)=\dot{\lambda}\left(\tau_{0}\right) \exp \left(\int_{\tau_{0}}^{\tau}-K(s) d s\right) \triangleq \dot{\lambda}\left(\tau_{0}\right) \Phi\left(\tau, \tau_{0}\right)
$$

Remark 1. The analysis is valid as long as the one-to-one correspondence holds between variables $(t, r, \tau)$. To this end, the range should be strictly decreasing with respect to time, i.e., $\dot{r}<0$. Defining a new variable $\eta=\sin \sigma$, the differential equation can be written as [31]

$$
\begin{aligned}
& \frac{d \eta}{d r} \triangleq \frac{K_{\eta}(r)}{r} \eta=\frac{N(r)-1}{r} \eta \\
& \frac{d \eta}{d \tau}=-K_{\eta}(\tau) \eta=-(K(\tau)+1) \eta
\end{aligned}
$$

From Eq. (10), it is clear that Assumption 1 holds whenever $|\eta|$ is strictly bounded by 1 , i.e.

$$
|\eta(\tau)|=\left|\eta_{0}\right| \exp \left(-\int_{\tau_{0}}^{\tau}(K(s)+1) d s\right)<1
$$

Note that $|\exp (x)|=\exp (x)>0$ for all $x \in \mathbb{R}$. By change of variable $\tau=\tau_{0}+x, x \leq 0$ into Eq. (11), the inequality condition can be equivalently expressed in terms of $K(\tau)$ as

$$
\int_{\tau_{0}}^{\tau_{0}+x} K(s) d s+x>\ln \left|\eta_{0}\right|, \quad \forall x \in[0, \infty)
$$

\section{Stability Analysis of Guidance Laws}

This section establishes a sufficient condition for the asymptotic convergence to a collision course that can be used as a new criterion applicable to cope with a wider class of guidance laws. We inspect how the guidance gain should be designed in order to adopt the extended stability theory of Theorem 1 in guidance problems. 


\section{A. Asymptotic Stability Criterion}

This section addresses the stability of $\dot{\lambda}$-dynamics. The standard Lyapunov stability theorem determines a sufficient condition of $K(\tau)$ to stabilise the the LOS rate given in Eq. (9) as

$$
K(\tau)>0, \quad \forall \tau \in\left[\tau_{0}, \infty\right)
$$

The positivity of $K(\tau)$ in the entire interval produces a monotonically converging pattern of the trajectory. Many guidance laws such as observability enhanced and evasive maneouvres include some intervals in which $K(\tau)$ is negative in modulating the trajectory. Using the properties of Theorem 2 , we can derive conditions to be satisfied by the associated averaged system while allowing $K(\tau)$ to be negative in some intervals. The following properties describe the criteria for system stabilization in this respect.

Theorem 2. Consider the engagement kinematics given by Eq. (7) defined with respect to the variable $\tau=-\ln r \in\left[\tau_{0}, \infty\right)$. The closed-loop system is asymptotically stable in $\tau$ if there exists a finite $T>0$ for all $\tau$ such that the following condition holds

$$
\int_{\tau}^{\tau+T} K(s) d s>0
$$

Proof. Consider a Lyapunov candidate function

$$
V(\tau)=\frac{1}{2} \dot{\lambda}(\tau)^{2}
$$

Using Eq. (9), the temporal difference of $V(\tau)$ between $\tau+T$ and $\tau$ gives

$$
\begin{aligned}
V(\tau+T)-V(\tau) & =\frac{\dot{\lambda}(\tau)^{2}}{2}\left((\Phi(\tau+T, \tau))^{2}-1\right) \\
& =V(\tau)\left(\exp \left(\int_{\tau}^{\tau+T}-2 K(s) d s\right)-1\right)
\end{aligned}
$$

By substituting Eq. (14) into (16), it can be proved that $V(\tau+$ $T)-V(\tau)<0$, which completes the proof.

There are several remarks on the stability criterion proposed in this study.

- (Physical meaning of $T$ ) $T$ physically implies a decreasing rate of the range in stabilising states. By the definition, $r_{1}$ corresponding to $\tau_{1}=\tau+T$ can be expressed as

$$
r_{1}=\exp \left(-\tau_{1}\right)=\exp (-\tau-T)=r_{0} \exp (-T) \triangleq \beta r_{0}
$$

where $\beta=\exp (-T) \leq 1$ for $T>0$. A sequence $\left\{r, \beta r, \beta^{2} r \cdots,\right\}$ is convergent as $\beta^{n} r \rightarrow 0$ for all $r \leq r_{0}$. A small value of $T$ tends to fortify the convergence of the system but also restricts the degree of the flexibility of the manoeuvre. In the extreme case of $T \rightarrow 0$, the integrand condition becomes $K(\tau)>0$, which is equivalent to the standard Lyapunov stability criterion. A large value of $T$ enlarges the size of the interval $[\tau, \tau+T]$, and $T$ tends to allow an increased degree of freedom in the choice of possible response patterns over the given interval.
- (Relevance with stability of averaged system) The average effect of the gain over the specific time interval is also positive when the condition of Theorem 3 holds, because

$$
K_{\text {avg }}=\frac{1}{T} \int_{\tau}^{\tau+T} K(s) d s>0
$$

This implies that the state $\dot{\lambda}$ tends to decrease in the average sense during the range interval $[r, \beta r]$.

- In choosing the parameter $T$ for guidance law design, a too large $T$ may cause violation of the assumption $\dot{r}<0$. Therefore, $T$ is only feasible as long as condition (12) holds. The feasible range of $x$ satsifying Eq. (12) can be obtained by solving

$$
\begin{array}{r}
\text { find minimum value } x=x(\bar{T}) \\
\text { s.t. } \quad\left(\int_{\tau_{0}}^{\tau_{0}+x} K(s) d s+x=\ln \left|\eta_{0}\right|\right)
\end{array}
$$

If $K$ is continuous in $\tau$, the upper bound $\bar{T}$ can be obtained by finding the extremum point $x(\bar{T})<\bar{T}$ that satisfies the following conditions

$$
K\left(\tau_{0}+x(\bar{T})\right)+1=0, \quad \int_{\tau_{0}}^{\tau_{0}+x(\bar{T})} K(\tau) d \tau+x(\bar{T})=\ln \left|\eta_{0}\right|
$$

- (Stronger stability criterion (14) for practical requirement) The asymptotic stability of the system is well explained in the $\tau$ domain, but the behaviour of the system near the interception may cause rapid manoeuvre or cause the high-frequency excitation in the inner loop. To handle such issues, it is desirable to design the gain $K(\tau)$ to satisfy the following condition.

$$
\begin{aligned}
K(\tau)>0 & \text { for } \quad \tau \geq-\ln r_{1} \\
\int_{\tau}^{\tau+T} K(s) d s>0 & \text { for } \quad-\ln r_{0} \leq \tau \leq-\ln r_{1}
\end{aligned}
$$

where $r_{1}$ is a specified value. Using Eq. (21), the trajectory can be modulated such that the closed-loop response shows the monotonic convergence in LOS rate near the end of the engagement while satisfying the condition in Theorem 2. The largest possible value of $T$ can be selected as $\bar{T}=\ln \left(r_{1} / r_{\text {miss }}\right)$, if $r$ decreasing below a certain threshold $r_{\text {miss }}$ is regarded as a successful interception. Note that the above condition does not imply the guidance law should switch at $r_{1}$. Instead, one can choose suitable design parameters in $K$ to make the guidance command consistently continuous in time.

\section{B. Stability Analysis of Existing Guidance Laws}

This section revisits existing guidance laws from the viewpoint of the extended stability theory discussed in Sec. III-A. Given a navigation gain $N(r)$, the problem considered in this study can be stated as follows.

Problem 1. Find a finite $T \geq 0$ such that the following condition holds for all $\tau$.

$$
\mathcal{F}(\tau ; T) \triangleq \int_{\tau}^{\tau+T} K(s) d s>0
$$


The following examples show how the extended stability theory enables analysis of the PNG variants that has remained impossible with the standard Lyapunov theory.

1) Adaptive Intermittent Manoeuvre Strategy: In this section, consider the AIM strategy [1], [3]. The central concept is to maintain the LOS rate within a predefined layer and achieve both information acquisition and LOS stabilisation by switching the navigation gain. The switching layers $\dot{\lambda}_{U}$ and $\dot{\lambda}_{L}$ are introduced as

$$
\dot{\lambda}_{U}=\mu_{U} r^{k}=\mu_{U} \exp (-k \tau), \quad \dot{\lambda}_{L}=\mu_{L} r^{k}=\mu_{L} \exp (-k \tau)
$$

where the design parameter $k$ is a positive constant. The guidance law for AIM is expressed as

$$
a_{A I M}=S_{l} N V_{M} \dot{\lambda}
$$

where $S_{l}$ is the switching sequence given by

$\mathcal{S}_{0}=\left\{\begin{array}{lll}1 & \text { if } & |\dot{\lambda}|>\dot{\lambda}_{L} \\ 0 & \text { if } & |\dot{\lambda}| \leq \dot{\lambda}_{L}\end{array} \quad, \mathcal{S}_{l}=\left\{\begin{array}{cll}1 & \text { if } & |\dot{\lambda}| \geq \dot{\lambda}_{U} \\ \mathcal{S}_{l-1} & \text { if } & \dot{\lambda}_{L} \leq|\dot{\lambda}| \leq \dot{\lambda}_{U} \\ 0 & \text { if } & |\dot{\lambda}| \leq \dot{\lambda}_{L}\end{array}\right.\right.$

Suppose that $\dot{\lambda}\left(\tau_{0}\right)$ is chosen within the layer $\left[\dot{\lambda}_{L}, \dot{\lambda}_{U}\right]$, and the algorithm is initialised with $S_{0}=0$. Then $\dot{\lambda}$ grows until it reaches the upper bound $\dot{\lambda}_{U}$. After while, the LOS rate shows a zig-zag pattern due to the on-off sequence. Substituting Eqs. (24) and (25) into Eq. (9), $\dot{\lambda}$-dynamics can be expressed as

$$
\frac{d \dot{\lambda}}{d \tau}=-K(\tau) \dot{\lambda}=\left\{\begin{array}{cc}
2 \dot{\lambda} & \tau_{0} \leq \tau \leq \tau_{1, \text { on }} \\
-(N-2) \dot{\lambda} & \tau_{i, \text { on }} \leq \tau \leq \tau_{i, \text { off }} \\
2 \dot{\lambda} & \tau_{i, \text { off }} \leq \tau \leq \tau_{i+1, \text { on }}
\end{array}\right.
$$

where $\tau_{i, o n}$ and $\tau_{i, o f f}$ correspond to the $i$-th incidents at which $\dot{\lambda}$ reaches the lower/upper bounds, respectively. Ref. [1] shows that the transition interval $\tau_{i, o f f}-\tau_{i, o n}$ and $\tau_{i+1, o n}-\tau_{i, o n}$ is consistent at every cycle. The ratio of transition $\alpha_{1}$ and $\alpha_{2}$ for cycle $i$ can be defined as

$$
\begin{aligned}
& \alpha_{1}=\tau_{i, o f f}-\tau_{i, \text { on }}=\frac{1}{N-2-k} \ln \left(\frac{\mu_{U}}{\mu_{L}}\right) \\
& \alpha_{2}=\tau_{i+1, \text { on }}-\tau_{i, \text { on }}=\frac{N}{(N-2-k)(2+k)} \ln \left(\frac{\mu_{U}}{\mu_{L}}\right)
\end{aligned}
$$

Note that the ratio is constant regardless of the cycle. The detailed derivation of Eq. (27) can be found in Ref. [1]. Alternating between increase and decrease in LOS rate (due to repeated switching of $S_{l}$ between 0 and 1) is necessary for observability enhancement. To trigger and maintain such fluctuating pattern until the end of engagement, Ref [1] suggests to choose a navigation gain bounded by parameter $k$ as

$$
N-2>k
$$

If the gain condition is violated, switching of manoeuvre takes place only once at the most, and the missile cannot perform the observability enhanced manoeuvre by AIM.

Now, we aim to show that the AIM guidance system satisfying condition (28) is guaranteed to be asymptotically stable. Note that the standard Lyapunov stability theorem cannot sufficiently explain the convergence with the 'zig-zag' pattern of the AIM response because the off sequence causes the growth of the LOS rate. Using the proposed stability criterion, the focus of the present analysis is to show the existence of $T$ satisfying Eq. (22). To this end, integration of $K(\tau)$ over the 'on' to 'on' sequence for cycle $i$ gives

$$
\begin{aligned}
\int_{\tau_{i, o n}}^{\tau_{i+1, o n}} K(\tau) d \tau & =\int_{\tau_{i, o n}}^{\tau_{i, o f f}} K(\tau) d \tau+\int_{\tau_{i, o f f}}^{\tau_{i+1, o n}} K(\tau) d \tau \\
& =(N-2) \alpha_{1}-2\left(\alpha_{2}-\alpha_{1}\right)=N \alpha_{1}-2 \alpha_{2} \\
& =\frac{N}{N-2-k} \frac{k}{2+k} \ln \left(\frac{\mu_{U}}{\mu_{L}}\right)
\end{aligned}
$$

It is obvious that Eq. (28) is positive when the navigation gain satisfies the condition in Eq. (29). Using the property, the asymptotic stability can be investigated for two cases. First, suppose that the manoeuvre mode is 'off', i.e., $K=-2$, at the current $\tau$. Then the duration $\Delta \tau$ until $\dot{\lambda}$ reaches $\dot{\lambda}_{U}(\tau)$ has the following relation:

$$
\dot{\lambda}(\tau) \exp (2 \Delta \tau)=\mu_{U} \exp (-k(\tau+\Delta \tau))
$$

$\Delta \tau$ can be calculated as

$$
\Delta \tau=-\frac{k}{k+2} \tau+\frac{1}{k+2} \ln \left(\frac{\mu_{U}}{\dot{\lambda}(\tau)}\right)
$$

Because $\mu_{L} \exp (-k \tau) \leq \dot{\lambda} \leq \mu_{U} \exp (-k \tau)$, the following inequality holds

$$
-\ln \mu_{U} \leq-k \tau-\ln \dot{\lambda}(\tau) \leq-\ln \mu_{L}
$$

From (32), it is easy to show that $\Delta \tau \leq \alpha_{2}-\alpha_{1}$. Now, consider $T$ as the transition ratio $\alpha_{2}$ as

$$
T=\alpha_{2}=\frac{N}{(N-2-k)(2+k)} \ln \left(\frac{\mu_{U}}{\mu_{L}}\right)
$$

$\mathcal{F}(\tau, T)$ can be expressed as

$$
\begin{aligned}
\mathcal{F}(\tau, T) & =\int_{\tau}^{\tau+T} K(s) d s \\
& =\int_{\tau}^{\tau+\Delta \tau}-2 d s+\int_{\tau+\Delta \tau}^{\tau+\Delta \tau+\alpha_{1}}(N-2) d s+\int_{\tau+\Delta \tau+\alpha_{1}}^{\tau+\alpha_{2}}-2 d s \\
& =-2 \Delta \tau+(N-2) \alpha_{1}-2\left(\alpha_{2}-\left(\Delta \tau+\alpha_{1}\right)\right) \\
& =N \alpha_{1}-2 \alpha_{2}>0
\end{aligned}
$$

Likewise, suppose that the manoeuvre mode is 'on', i.e., $K=$ $N-2$, at the current $\tau$. The duration $\Delta \tau_{2}$ until $\dot{\lambda}$ reaches the lower switching boundary $\dot{\lambda}_{L}(\tau)$ has the following relation:

$$
\dot{\lambda}(\tau) \exp \left(-(N-2) \Delta \tau_{2}\right)=\mu_{L} \exp \left(-k\left(\tau+\Delta \tau_{2}\right)\right.
$$

$\Delta \tau_{2}$ can be obtained as

$$
\Delta \tau_{2}=\frac{k}{N-2-k} \tau+\ln \left(\frac{\dot{\lambda}(\tau)}{\mu_{L}}\right)
$$

From (32), $\Delta \tau_{2} \leq \alpha_{1}$. Then it can be shown that $\mathcal{F}(\tau, T)$ is greater than zero as

$$
\begin{aligned}
\mathcal{F}(\tau, T) & =\int_{\tau}^{\tau+T} K(s) d s \\
& =\int_{\tau}^{\tau+\Delta \tau_{2}}(N-2) d s+\int_{\tau+\Delta \tau_{2}}^{\tau+\Delta \tau_{2}+\left(\alpha_{2}-\alpha_{1}\right)}-2 d s+\int_{\tau+\Delta \tau_{2}+\left(\alpha_{2}-\alpha_{1}\right)}^{\tau+\alpha_{2}}(N-2) d \tau \\
& =(N-2) \Delta \tau_{2}-2\left(\alpha_{2}-\alpha_{1}\right)+(N-2)\left(\alpha_{1}-\Delta \tau_{2}\right)=N \alpha_{1}-2 \alpha_{2}>0
\end{aligned}
$$

By Theorem 2, it can be shown that $N>2+k$ ensures the stability of the equilibrium $\dot{\lambda}=0$ with $T=\alpha_{2}$. In summary, 
the extended stability criterion ensures the non-monotonic asymptotic convergence of LOS rate regardless of the current manoeuvre mode at a given instance, provided that the gain satisfies the condition of Eq. (28).

2) Observability-Enhancement Optimal Guidance:

Observability-enhancement optimal guidance (OEOG) law [6] was designed to enhance the target observability in passive homing missiles in the optimal control framework. The guidance command is converted to a PNG form, where the navigation gain is given by

$$
\begin{aligned}
N & =\omega\left(\frac{r}{V}\right)^{N_{f}} \cot \left(\frac{\omega}{N_{f}}\left(\frac{r}{V}\right)^{N_{f}}\right) \\
& =\frac{\omega}{V^{N_{f}}} \exp \left(-N_{f} \tau\right) \cot \left(\frac{\omega}{N_{f} V^{N_{f}}} \exp \left(-N_{f} \tau\right)\right) \\
& \triangleq N_{f} h(z)=N_{f} z \cot z
\end{aligned}
$$

where $\omega>0$ and $N_{f}>2$ are the design parameters, and $z(\omega, \tau)=\frac{\omega}{N_{f}}\left(\frac{r}{V}\right)^{N_{f}}=\frac{\omega}{N_{f} V^{N_{f}}} \exp \left(-N_{f} \tau\right)$ is the auxiliary variable dependent on $\omega$ and $\tau$. It is assumed that $\omega$ is properly chosen so that $z(\omega, \tau)<\pi$ to prevent singularity of $N$. For ease of notation, $\omega$ and $\tau$ in $z$ are omitted. Now, let us analyse the stability of OEOG under the extended stability criterion. The stability analysis aims to show the existence of a finite $T>0$ for all $\tau$ that satisfies the following inequality with $\mathcal{F}(\tau ; T)$ :

$$
\begin{aligned}
\mathcal{F}(\tau ; T) & =\int_{\tau}^{\tau+T}\left(N_{f} z(\omega, s) \cot z(\omega, s)-2\right) d s \\
& =\int_{z(\tau)}^{z(\tau+T)}-\cot (z) d z-\int_{0}^{T} 2 d \tau \\
& =-\ln \left(\sin \left(\frac{\omega}{N_{f}}\left(\frac{r}{V}\right)^{N_{f}} \exp \left(-N_{f} T\right)\right)\right)+\ln \left(\sin \left(\frac{\omega}{N_{f}}\left(\frac{r}{V}\right)^{N_{f}}\right)\right)-2 T>0
\end{aligned}
$$

Suppose that $T$ satisfies

$$
T \geq \frac{1}{N_{f}-2} \ln \left(\frac{\frac{\omega}{N_{f}}\left(\frac{r_{0}}{V}\right)^{N_{f}}}{\sin \left(\frac{\omega}{N_{f}}\left(\frac{r_{0}}{V}\right)^{N_{f}}\right)}\right)
$$

Since $0 \leq z<\pi, \sin z<z$ and $-\ln (\sin z)>-\ln z$, we have

$$
\begin{aligned}
\mathcal{F}(\tau ; T) & \geq-\ln \left(\frac{\omega}{N_{f}}\left(\frac{r}{V}\right)^{N_{f}} \exp \left(-N_{f} T\right)\right)+\ln \left(\sin \left(\frac{\omega}{N_{f}}\left(\frac{r}{V}\right)^{N_{f}}\right)\right)-2 T \\
& =\left(N_{f}-2\right) T+\ln \left(\frac{\sin \left(\frac{\omega}{N_{f}}\left(\frac{r}{V}\right)^{N_{f}}\right)}{\frac{\omega}{N_{f}}\left(\frac{r}{V}\right)^{N_{f}}}\right)
\end{aligned}
$$

Because $z \leq z_{0}<\pi$ and $\frac{z}{\sin z} \leq \frac{z_{0}}{\sin z_{0}}$, Eqs. (40) and (41) yield

$$
\begin{aligned}
\mathcal{F}(\tau ; T) & \geq\left(N_{f}-2\right) T+\ln \left(\frac{\sin \left(\frac{\omega}{N_{f}}\left(\frac{r}{V}\right)^{N_{f}}\right)}{\frac{\omega}{N_{f}}\left(\frac{r}{V}\right)^{N_{f}}}\right) \\
& \geq \ln \left(\frac{\frac{\omega}{N_{f}}\left(\frac{r_{0}}{V}\right)^{N_{f}}}{\sin \left(\frac{\omega}{N_{f}}\left(\frac{r_{0}}{V}\right)^{N_{f}}\right)}\right)-\ln \left(\frac{\frac{\omega}{N_{f}}\left(\frac{r}{V}\right)^{N_{f}}}{\sin \left(\frac{\omega}{N_{f}}\left(\frac{r}{V}\right)^{N_{f}}\right)}\right)>0
\end{aligned}
$$

Therefore, the existence of $T$ satisfying Eq. (40) implies that the OEOG asymptotically stabilises the LOS rate according to Theorem 2. It is worth noting that the parameters $N_{f}$ and $\omega$ are properly selected to satisfy Eq. (12) in Remark 1. Using Eq.
(20), an extreme point $x$ and an upper bound $\bar{\omega}$ that comprises the feasible range $\Omega_{\text {extended, } \mathcal{F}>0}=[0, \bar{\omega}]$ can be obtained by solving the algebraic equation:

$$
\begin{aligned}
& K\left(\tau_{0}+x\right)+1=\omega\left(\frac{r_{0}}{V}\right)^{N_{f}} \exp \left(-N_{f} x\right) \cot \left(\frac{\omega}{N_{f}}\left(\frac{r_{0}}{V}\right)^{N_{f}} \exp \left(-N_{f} x\right)\right)-1=0 \\
& \mathcal{F}\left(\tau_{0} ; x\right)=-\ln \left(\sin \left(\frac{\omega}{N_{f}}\left(\frac{r_{0}}{V}\right)^{N_{f}} \exp \left(-N_{f} x\right)\right)\right)+\ln \left(\sin \left(\frac{\omega}{N_{f}}\left(\frac{r_{0}}{V}\right)^{N_{f}}\right)\right)-x=\ln \left|\eta_{0}\right|
\end{aligned}
$$

Note that Eq. (43) should be sloved numerically to obtain $x=$ $x^{*}$ and $\omega=\bar{\omega}$. To this end, let us define another auxiliary variable $a$ as

$$
a=\exp (x) \geq 1
$$

Equation (43) can be expressed in terms of $z$ and $a$ as

$$
\begin{aligned}
& h(z): N_{f} z \cot z-1=0 \\
& f_{2}(a ; z):-\ln \left(\sin \left(z^{*}\right)\right)+\ln \left(\sin \left(z^{*} a^{N_{f}}\right)\right)-\ln a=\ln \left|\eta_{0}\right|
\end{aligned}
$$

Note that $h(z)=z \cot z$ is one-to-one function from $D=$ $\left(0, z_{0}\right] \in(0, \pi)$ to $(-\infty, 1)$. Thus, Eq. (45a) always has the solution in the interval such that $h\left(z^{*}\right)=\frac{1}{N_{f}}$. Letting $z^{*}=h^{-1}\left(\frac{1}{N_{f}}\right)$, Eq. (45b) is equivalent to

$$
\frac{\sin \left(z^{*} a^{N_{f}}\right)}{a}=\sin \left(z^{*}\right)\left|\eta_{0}\right|
$$

It can be shown that $f_{2}(a)=\sin \left(z^{*} a^{N_{f}}\right) / a$ is one-to-one correnspondence, and it is bounded as $f_{2} \in\left[0, \sin \left(z^{*}\right)\right]$ for $a \in\left[1, \pi /\left(z^{*}\right)\right]$ because $\mathrm{f}_{2}(a) / d a \leq 0$. From mean value theorem, there exists $a^{*}$ satsifying $f_{2}\left(a^{*}\right)=\sin \left(z^{*} a^{* N_{f}}\right) / a^{*}=$ $\sin \left(z^{*}\right)\left|\eta_{0}\right|$. Using the solution $z^{*}$ and $a^{*}, x$ and $\omega$ can be calculated as

$$
\omega=N_{f} z^{*} a^{N_{f}}\left(\frac{V}{r_{0}}\right)^{N_{f}}, \quad x=\ln a^{*}
$$

Hence, feasible range of $\omega$ and $\mathrm{T}$ using extended stability criteria is obtained as

$$
\begin{aligned}
& \omega \in \Omega_{\text {extended, } \mathcal{F}>0}=\left[0, N_{f} z^{*} a^{N_{f}}\left(\frac{V}{r_{0}}\right)^{N_{f}}\right], \\
& T \in\left[0, \frac{1}{N_{f}-2} \ln \left(\frac{z^{*} a^{N_{f}}}{\sin \left(z^{*} a^{N_{f}}\right)}\right)\right]
\end{aligned}
$$

\section{Design of a Weaving Guidance Law with Guaranteed Non- monotonic Convergence}

This section presents a simple example showing how to design a new range-varying navigation gain based on the stability criterion. Under the PNG structure, consider the guidance gain $N(\tau)$ given by the following form:

$$
K=N_{\text {base }}+N_{\text {pert }}(\tau)-2=N-2+N_{\text {pert }}(\tau)
$$

Then, the sufficient condition for asymptotic stability of the guidance system is existence of $T$ such that

$$
\int_{\tau}^{\tau+T}\left\{(N-2)+N_{\text {pert }}(s)\right\} d s=(N-2) T+\int_{\tau}^{\tau+T} N_{\text {pert }}(s) d s>0, \quad \forall \tau \in\left[\tau_{0}, \infty\right)
$$

Now, let us consider the following guidance problem. 
Problem 2. Suppose that the perturbation term $N_{\text {pert }}$ is parameterised by $\mathbf{p}$, and the length of the interval $T$ with which the sufficient condition for stable convergence should hold. The guidance problem under consideration is to find proper guidance parameters $\mathbf{p}$ associated with $N_{\text {pert }}(\tau ; \mathbf{p})$ by satisfying the following condition to ensure the asymptotic stability.

$$
\int_{\tau}^{\tau+T} N_{\text {pert }}(s ; \mathbf{p}) d s>-(N-2) T
$$

In this study, a weaving manoeuvre is considered to perform dual-control guidance or evasive manoeuvre during the terminal phase. The perturbation gain includes an oscillatory component to produce the manoeuvre and reduces its effect near the interception to prevent any conflict with the baseline PPNG. To this aim, consider the navigation gain given by the following form

$N_{\text {pert }}\left(\tau ; A_{w}, \omega\right)=A_{w} \sin \left(\omega \frac{r}{r_{0}}\right)=A_{w} \sin \left(\omega \exp \left(-\left(\tau-\tau_{0}\right)\right)\right)$

where the magnitude $A_{w}$ and frequency $\omega$ denote the parameters to be determined for stability. The integral of $N_{\text {pert }}$ is calculated as

$$
\int_{\tau}^{\tau+T} N_{\text {pert }}(s) d s=-A_{w}\left(\operatorname{Si}\left(\omega e^{-(\tau+T)}\right)-\operatorname{Si}\left(\omega e^{-\tau}\right)\right)
$$

where $\operatorname{Si}(x)=\int_{0}^{x} \frac{\sin s}{s} d s$. By substituting Eq. (53) into (51), the parameters $A_{w}$ and $\omega$ must satisfy the following inequality for any $\tau \geq \tau_{0}$

$$
-A_{w}\left(\operatorname{Si}\left(\omega e^{-\left(\tau-\tau_{0}+T\right)}\right)-\operatorname{Si}\left(\omega e^{-\left(\tau-\tau_{0}\right)}\right)\right)>-(N-2) T
$$

Let $\alpha=\omega e^{-\left(\tau-\tau_{0}\right)}<\omega$ and $\beta=\exp (-T)<1$ be auxiliary variables. The partial derivative of the right-hand side of Eq. (53) with respect to $\alpha$ is

$$
\frac{\partial\left(-A_{w}(\operatorname{Si}(\alpha \beta)-\operatorname{Si}(\alpha))\right)}{\partial \alpha}=-A_{w}\left(\frac{\sin (\alpha \beta)-\sin \alpha}{\alpha}\right)
$$

For $\omega>0$ and $A_{w}<0$, Eq. (54) takes its minimum value at $\alpha=\frac{\pi}{\beta+1}$. Substituting the extreme point into Eq. (54) gives

$$
-\frac{(N-2) T}{\operatorname{Si}\left(\frac{\exp (T)}{\exp (T)+1} \pi\right)-\operatorname{Si}\left(\frac{\pi}{\exp (T)+1}\right)}<A_{w}<0
$$

Equation (56) implies that $A_{w}$ is the only parameter associated with $T$. A large value of $T$ leads to a large magnitude $A_{w}$. Also, the greater the $\omega$ value is, the more oscillatory behaviour the guidance law exhibits. High-frequency oscillations should be avoided because an abrupt change in the guidance command may lead to potential instabilities. Considering the range $r_{1}$ of Eq. (22) in which the strict convergence is assured, the frequency can be bounded by

$$
\omega \frac{r}{r_{0}}<\frac{\pi}{2}, \quad A_{w} \sin \left(\omega \frac{r}{r_{0}}\right)+N>2, \quad \text { for all } r \leq r_{1}
$$

Note that $A_{w}<0$ obtained from Eq. (56). By substituting $r=r_{1}$ into $\mathrm{Eq}$ (57), the feasible range of $\omega$ can be obtained as

$$
0<\omega \leq \min \left(\frac{\pi r_{0}}{2 r_{1}}, \frac{r_{0}}{r_{1}} \sin ^{-1}\left(\frac{2-N}{A_{w}}\right)\right)
$$

It is worth noting that the inequality condition in Eq. (58) carries a slight degree of conservatism stemming from Assumption 1 which requires the distance to decrease monotonically to zero even though the LOS rate may exhibit non-monotonic behaviours.

\section{Simulation Results}

This section performs numerical simulations to verify analysis results based on the presented stability criterion. We consider guidance laws presented in Sec. III-B and III-C to demonstrate the benefits of using the extended stability criterion in relation to the choice of design parameters. The simulation is performed in MATLAB environment, where a fixed-time solver is used for numerical integration with $\Delta t_{\text {sim }}=5 \cdot 10^{-4} \mathrm{~s}$. The simulation is terminated if $r$ is less than $0.1 \mathrm{~m}$. A second-order lag model $\frac{a(s)}{a_{c}(s)}=\frac{\omega_{n}^{2}}{s^{2}+2 \zeta \omega_{n} s+\omega_{n}^{2}}$ with magnitude limiter $|a| \leq a_{\max }$ is included in simulation for autopilot response, $\zeta=0.8$ is the damping ratio, and $\omega_{n}$ is the natural frequency which is set to $\omega_{n}=2 \zeta / 0.05=32 / \mathrm{sec}$ [35]. Other simulation parameters are summarised as follows

$$
\begin{aligned}
& \left(X_{m}, Y_{m}\right)=(0,1,000 m),\left(X_{t}, Y_{t}\right)=(10,000 m, 0) \\
& V_{M}=300 \mathrm{~m} / \mathrm{s}, \gamma_{0}=30 \mathrm{deg}, N_{\text {base }}=3, a_{\max }=100 \mathrm{~m} / \mathrm{s}^{2}
\end{aligned}
$$

\section{A. Performance of AIM Strategy [1]}

In this simulation case, AIM is considered with different design parameters $k$ and initial conditions $\sigma_{0}$. In cases 1-1 and $1-2$, the parameter $k$ is selected to satisfy the condition in Eq. (28). $\mu_{L}$ and $\mu_{U}$ are chosen as $\mu_{L}=\left|\dot{\lambda}_{0}\right| / r_{0}^{k}=0.5 V_{M} / r_{0}^{1.2}$ and $\mu_{U}=1.4 \mu_{L}=0.7 V_{M} / r_{0}^{1.2}$ so that the AIM starts the simulation on the lower switching layer. For a comparison study, case 1-3 uses $k=1.2$ which does not satisfy the condition (28). Note that the repeated switching sequence may involve chattering near the interception. To prevent this phenomenon, the lower switching layer is truncated to zero when $r<r_{1}=300 \mathrm{~m}$ [3]. The following functional is also introduced to discuss the performance of AIM strategy.

$$
\mathcal{F}_{0}\left(\tau ; \tau_{0}\right)=\int_{\tau_{0}}^{\tau_{0}+\tau} K(s) d s
$$

By definition, $\mathcal{F}(\tau ; T)=\mathcal{F}_{0}\left(\tau+T ; \tau_{0}\right)-\mathcal{F}_{0}\left(\tau ; \tau_{0}\right)$.

Figure 2 shows the simulation results for AIM strategy. The AIM allows to switch the navigation gain while regulating the variables $\sigma$ and $\dot{\lambda}$. As shown in Fig. 2-(b), the Lyapunov candidate function tends to converge to zero over $\tau$. Notably, $V$ increases in some intervals because of the 'off' sequence, which is not acceptable in the standard Lyapunov stability theorem. On the other hand, using the extended stability theorem addressed in this study, $\mathcal{F}(\tau, T)>0$ implies that $V(\tau)$ is less than $V(\tau+T)$ and eventually regulates to zero. Note that $\mathcal{F}$ is kept constant, meaning that the decrement is consistent regardless of $\tau$. Additionally, there exists a relationship between the Lyapunov function and $\mathcal{F}_{0}$ in that the Lyapunov function is less than the initial value when $\mathcal{F}_{0}$ is positive.

In case 1-2, the simulation shows similar results with case $1-1$, meaning that the asymptotic stability holds for different 


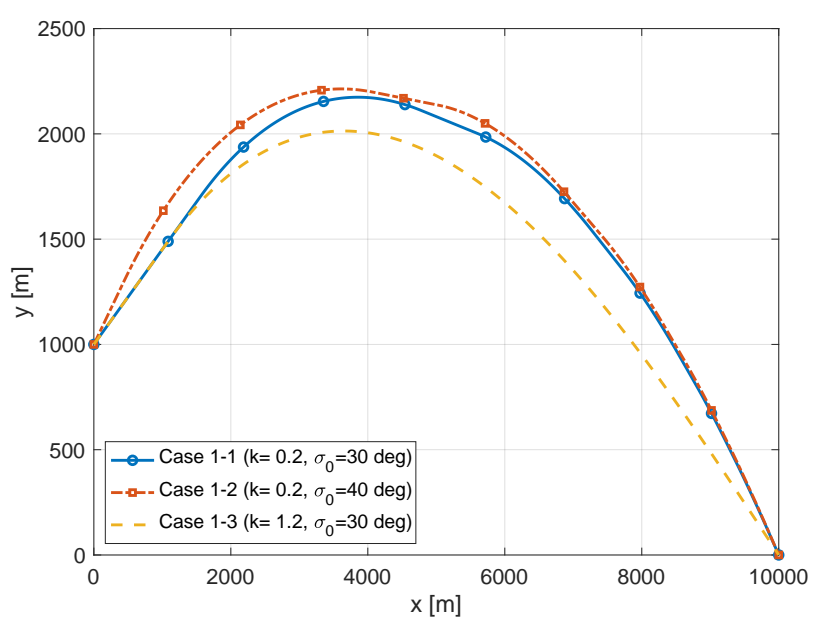

(a) Trajectory
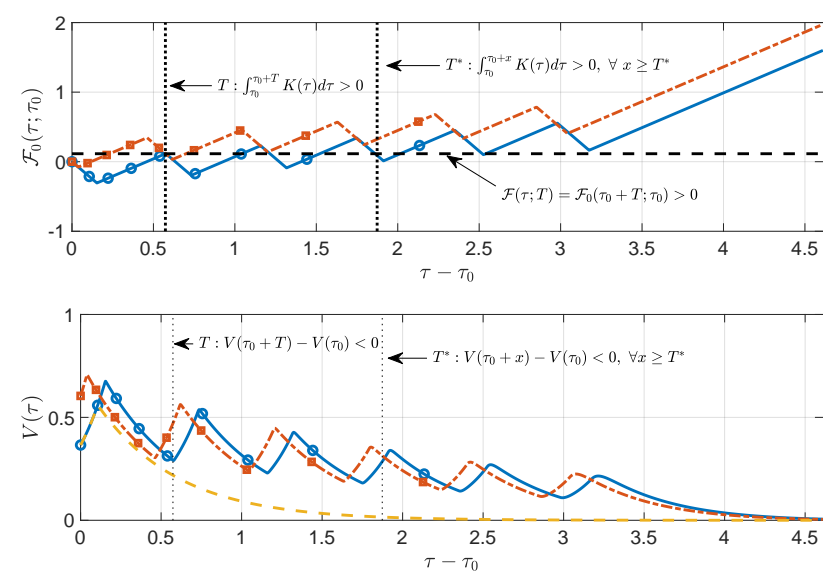

(c) $\mathcal{F}_{0}\left(\tau ; \tau_{0}\right)$ and $\mathrm{V}(\tau)$
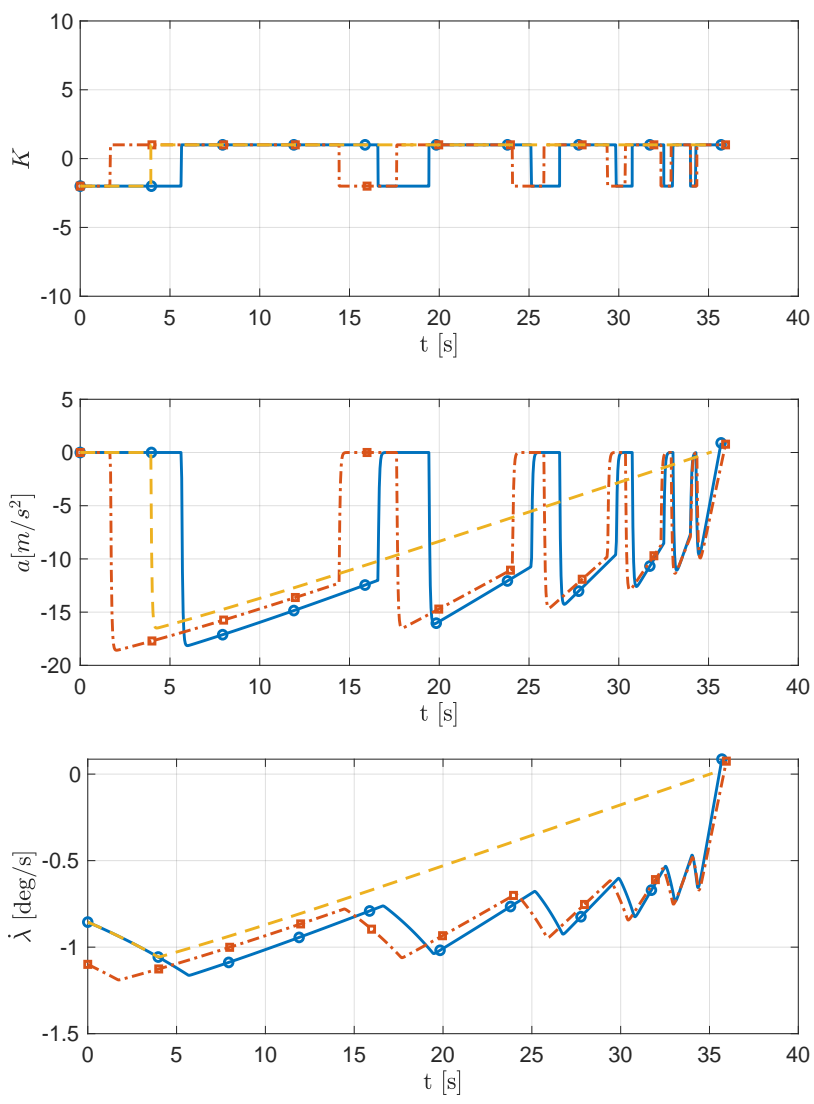

(b) Gain $K$, Acceleration, and LOS rate

Fig. 2: Simulation Results for Case 1: AIM Strategy [1]

initial value when $k$ is selected to satisfy the stability condition $N>k+2$. In case $1-3$, where the pair $(N, k)$ violates the condition Eq. (28), missile completes the interception but does not perform any observability enhancing manoeuvre as intended in the AIM strategy because the guidance gain is switched only once and maintains PPNG until the end of interception. The closed-loop response does not exhibit any instability phenomenon. The result indicates that satisfying Eq. (28) serves as a requirement to perform the repeated AIM manoeuvre for enhanced observability rather than a necessary condition for stability. The stability criterion is inherently a sufficient condition for stability rather than a full characterisation of the region of attraction. Nevertheless, the proposed stability criterion is valuable in that satisfaction of the requirement regarding the gain design guarantees closedloop stability, the argument which has been unavailable with the standard Lyapunov stability theory.

\section{B. Performance of Observability-Enhanced Optimal Guidance [6]}

Simulation case 2 aims to demonstrate with the numerical example using OEOG that the relaxed stability criterion based on Theorem 2 extends the feasible range of the design parameter for guaranteed asymptotic stability. For this purpose, the feasible range of $\omega$ based on the standard stability criterion is first obtained. The navigation gain of Eq. (38) satisfies the following properties

$$
\begin{array}{ll}
\frac{\partial h(z)}{\partial z}=\cot z-z \csc ^{2} z<0 & \text { for } 0 \leq z<\pi \\
\frac{\partial N(\tau)}{\partial \tau}=-N_{f}^{2} z \frac{\partial h(z)}{\partial z}>0 & \text { for } \tau_{0} \leq \tau<\infty
\end{array}
$$

By substituting $z\left(\tau_{0}\right)=\frac{\omega}{N_{f}}\left(\frac{r_{0}}{V}\right)^{N_{f}}$ into Eqs. (38) and (13), the stability criterion in terms of $\omega$ can be obtained as

$$
K(z)=N-2>\omega\left(\frac{r_{0}}{V}\right)^{N_{f}} \cot \left(\frac{\omega}{N_{f}}\left(\frac{r_{0}}{V}\right)^{N_{f}}\right)-2>0
$$

Equivalently, the feasible range $\Omega_{\text {standard, } K>0}$ can be determined as

$$
\Omega_{\text {standard }, K>0}=\left[0, N_{f}\left(\frac{V}{r_{0}}\right)^{N_{f}} h^{-1}\left(\frac{2}{N_{f}}\right)\right]
$$

where $h^{-1}(x)$ is the inverse function of $h(x)=x \cot x$. By substituting the simulation parameters into Eqs. (48) and (62), the feasible range of $\omega$ guaranteeing asymptotic stability based 
on the extended and the standard stability criteria can be calculated as follows.

$\Omega_{\text {extended, } \mathcal{F}>0}=[0,0.00190], \quad \Omega_{\text {standard }, K>0}=[0,0.00077]$

It is shown that there exists an inclusive relation between the feasible ranges as $\Omega_{\text {standard, } K>0} \subset \Omega_{\text {extended, } \mathcal{F}>0}$. Likewise, the feasible range of $T$ can be calculated as $0 \leq T \leq 1.2413$ from Eq. (48).

TABLE I: Simulation Parameter for Case 2

\begin{tabular}{lcccccc}
\hline \hline Case & $2-1$ & $2-2$ & $2-3$ & $2-4$ & $2-5$ & $2-6$ \\
$\omega$ & $0.7 \mathrm{E}-4$ & $1.4 \mathrm{E}-4$ & $1.9 \mathrm{E}-4$ & $1.901 \mathrm{E}-4$ & $1.905 \mathrm{E}-4$ & $2.0 \mathrm{E}-4$ \\
$T$ & 0 & 0.5791 & 1.2395 & 1.2413 & 1.2487 & 1.4406 \\
\hline \hline
\end{tabular}

Six examples are considered with different values of $\omega$ for comparative study. The parameters and the corresponding $T$ is summarised in Table I. Note that $\omega$ for case 2-1 lies in the feasible range $\Omega_{\text {standard, } K>0}$, and $\omega$ for cases $2-5$ and 26 is assigned outside the feasible range. Figure 3 shows the simulation result. The missiles for cases 2-1 to 2-4 achieve successful interception. For case $2-1$, the positive gain $K$ leads to the monotonic convergence to the collision course as shown in Fig. 3-(b). When $\omega$ is chosen in the feasible range (cases 2-2, 2-3, and 2-4), the gain $K$ takes a negative value at the beginning due to the observability enhancing manoeuvre, which increases the Lyapunov function in this period, as shown in Figs. 3-(b) and 3-(c). Since T obtained by Eq. (48) lie in the feasible range as shown in Table $1, \mathcal{F}_{0}$ is kept positive after $\tau \geq \tau_{0}+T$, and $V(\tau)$ consistently converges to zero as shown in Fig. 3-(c). It implies that the asymptotic convergence to the collision course is assured in the context of extended stability theory. It is also observed that the trajectory is elongated as the $\omega$ is selected as the closest to the extreme value. Any larger $T$ in the stable range permits a longer period of the increase LOS rate and thus elongates the path. It is noted that a large $\omega$ as shown in cases 2-5 and 2-6 causes an abrupt manoeuvre and deviates from the collision course when $\omega$ is selected outside the feasible range. The infeasible $T$ cannot ensure the asymptotic convergence to the collision course, and the missile fails to intercept the target.

\section{Performance of Guidance Law with Guaranteed Non- monotonic Convergence}

In this simulation, the weaving guidance law designed in Sec. III-C is considered. Three cases of the parameter sets are considered to demonstrate the performances with the stability perspective. For case $3-1, A_{w}$ and $\omega$ are selected to satisfy the criterion based on the standard Lyapunov stability theory. For the others, $A_{w}$ is calculated by a specified $T=4$, and different values of $\omega$ are assigned within the range obtained in Eq. (58) as follows.

$$
A_{w}=-\frac{(N-2) T}{\operatorname{Si}\left(\frac{\exp (T)}{\exp (T)+1} \pi\right)-\operatorname{Si}\left(\frac{\pi}{\exp (T)+1}\right)}+0.01=-2.22, \quad \omega \in\left[0, \frac{r_{0}}{500} \sin ^{-1}\left(\frac{2-N}{A_{w}}\right)\right]=[0,9.399]
$$

Figure 4 shows the simulation results. Missiles for all cases successfully intercept the target; meanwhile $\dot{\lambda}$ and $\sigma$ exhibit oscillatory response while converging to zero. As shown in case $3-1, A_{w}$ is assigned for a small value to keep $K$ positive, and therefore the perturbation effect is insignificant. When $A_{w}$ is selected considering the extended stability theorem, it amplifies the magnitude of oscillation. The gain $K(\tau)$ alternates to a negative value, $\mathcal{F}$ keeps positive for all interval to ensure stability. $\omega$ contributes to the oscillation frequency in $K$, which is fortified as it increases. The responses can be explained along with stability. This result indicates that the choice of $A_{w}$ and $\omega$ in the design process can ensure the asymptotic stability of the system while exploiting the increase of the Lyapunov function in some intervals.

\section{Conclusion}

This study presented a new stability criterion for proportional navigation guidance laws with range-varying navigation gain. The stability criterion does not demand the time derivative of the Lyapunov function to be strictly negative.The proposed criterion allows even increase of the Lyapunov function in some intervals while ensuring overall asymptotic stability. This stability condition is expressed in terms of the integral of navigation gain corresponding to the Lyapunov function variation. The length of the interval for the integral represents the degree of stability under which the guidance command tolerates the increase in the Lyapunov function and stabilises asymptotically. The stability condition suggested in this study applies to a wider class of guidance laws including those involving weaving manoeuvres. This analysis can be utilised to design new guidance schemes, e.g., guidance with evasive and observability-enhanced manoeuvres. The relaxed stability criterion may also provide a useful insight towards other control problems that demand dual-control approaches.

\section{REFERENCES}

[1] H.-I. Lee, M.-J. Tahk, and B.-C. Sun, "Practical dual-control guidance using adaptive intermittent maneuver strategy," Journal of Guidance, Control, and Dynamics, vol. 24, no. 5, pp. 1009-1015, 2001.

[2] M. G. Seo and M. J. Tahk, "Observability analysis and enhancement of radome aberration estimation with line-of-sight angle-only measurement," IEEE Transactions on Aerospace and Electronic Systems, vol. 51, no. 4, pp. 3321-3331, 2015.

[3] D. Lee, M.-J. Tahk, and C.-H. Lee, "Optimal threshold of intermittent maneuver for target observability improvement," International Journal of Aeronautical and Space Sciences, pp. 1-12, 2020.

[4] D. G. Hull, J. L. Speyer, and C. Y. Tseng, "Maximum-information guidance for homing missiles," Journal of Guidance, Control, and Dynamics, vol. 8, no. 4, pp. 494-497, 1985.

[5] D. G. Hull, J. L. Speyer, and D. B. Burris, "Linear-quadratic guidance law for dual control of homing missiles," Journal of Guidance, Control, and Dynamics, vol. 13, no. 1, pp. 137-144, 1990.

[6] S. He, H.-S. Shin, W.-S. Ra, and A. Tsourdos, "Observabilityenhancement optimal guidance law," in 2019 Workshop on Research, Education and Development of Unmanned Aerial Systems, Cranfield, UK, November 2019.

[7] R. J. Casler Jr, "Dual-control guidance strategy for homing interceptors taking angle-only measurements," Journal of Guidance and Control, vol. 1, no. 1, pp. 63-70, 1978.

[8] M.-J. Tahk, H. Ryu, and E.-J. Song, "Observability characteristics of angle-only measurement under proportional navigation," in 34th SICE Annual Conference, Hokkaido, Japan, July 1995.

[9] T. L. Song and T. Y. Um, "Practical guidance for homing missiles with bearings-only measurements," IEEE Transactions on Aerospace and Electronic Systems, vol. 32, no. 1, pp. 434-443, 1996.

[10] T.-H. Kim, C.-H. Lee, and M.-J. Tahk, "Time-to-go polynomial guidance with trajectory modulation for observability enhancement," IEEE Transactions on Aerospace and Electronic Systems, vol. 49, no. 1, pp 55-73, 2013. 


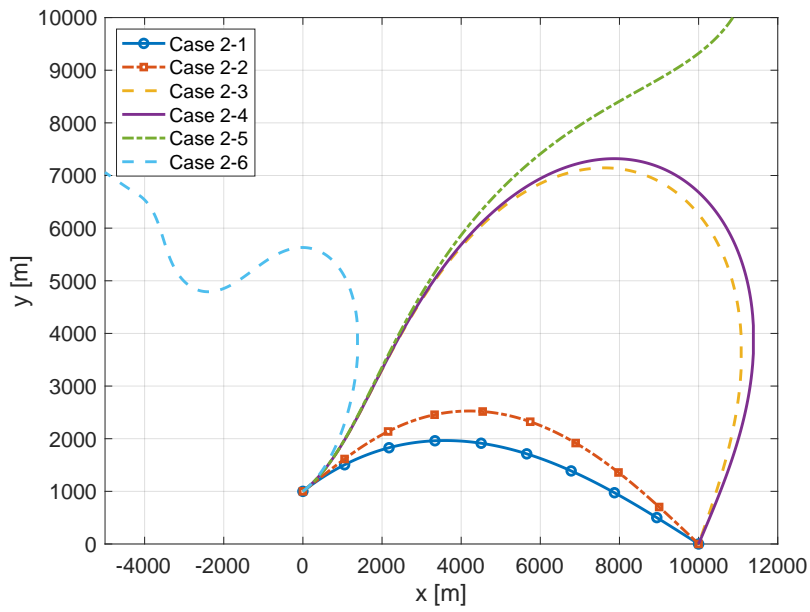

(a) Trajectory
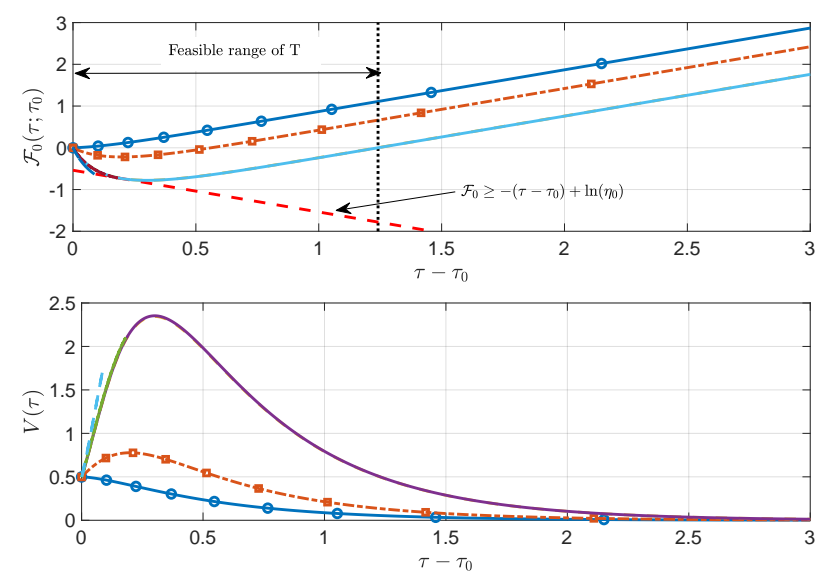

(c) $\mathcal{F}_{0}\left(\tau ; \tau_{0}\right)$ and $\mathrm{V}(\tau)$
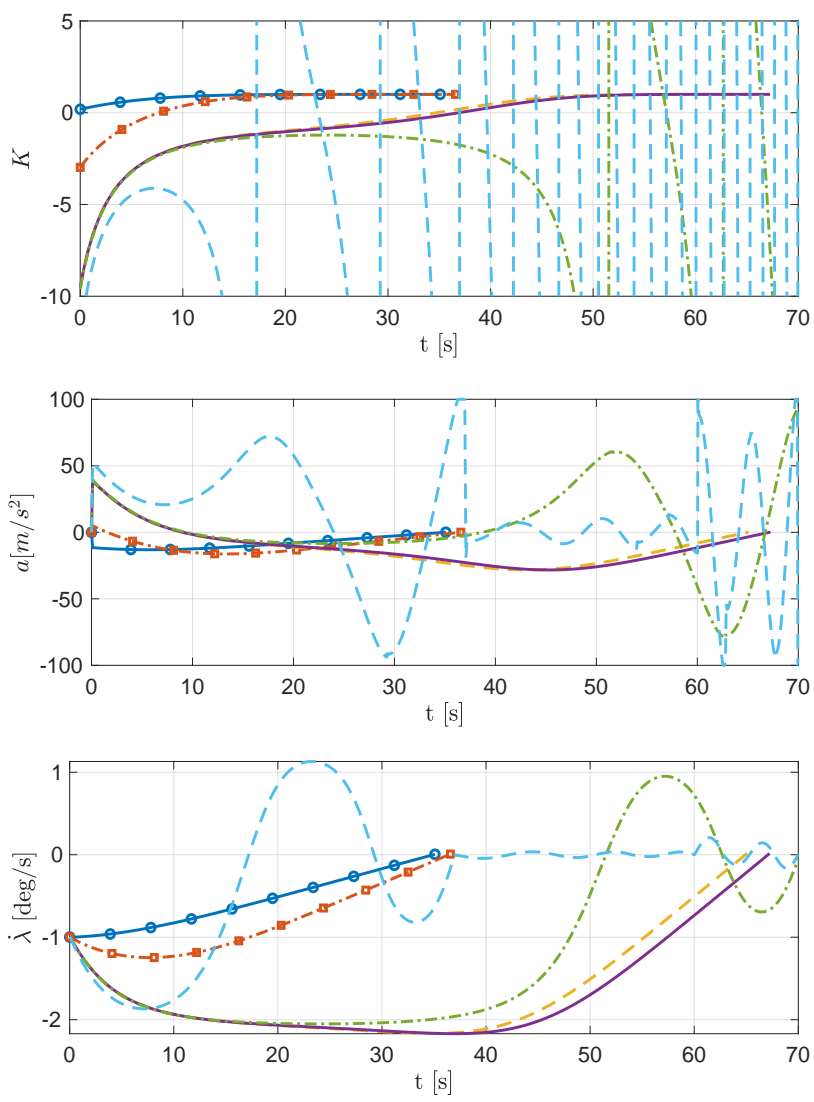

(b) Gain $K$, Acceleration, and LOS rate

Fig. 3: Simulation Results for Case 2 : OEOG [6]

[11] C. H. Lee, T. H. Kim, and M. J. Tahk, "Biased png for target observability enhancement against nonmaneuvering targets," IEEE Transactions on Aerospace and Electronic Systems, vol. 51, no. 1, pp. 2-17, 2015.

[12] H. I. Lee, H. S. Shin, and A. Tsourdos, "Weaving guidance for missile observability enhancement," in 20th IFAC World Congress, Toulouse, France, July 2017.

[13] Y.-H. Kim, C.-K. Ryoo, and M.-J. Tahk, "Guidance synthesis for evasive maneuver of anti-ship missiles against close-in weapon systems," IEEE Transactions on Aerospace and Electronic Systems, vol. 46, no. 3, pp. 1376-1388, 2010.

[14] C.-H. Lee, J.-I. Lee, and M.-J. Tahk, "Sinusoidal function weighted optimal guidance laws," Proceedings of the Institution of Mechanical Engineers, Part G: Journal of Aerospace Engineering, vol. 229, no. 3, pp. 534-542, 2015.

[15] J.-I. Lee and C.-K. Ryoo, "Impact angle control law with sinusoidal evasive maneuver for survivability enhancement," International Journal of Aeronautical and Space Sciences, vol. 19, no. 2, pp. 433-442, 2018.

[16] S. Brierley and R. Longchamp, "Application of sliding-mode control to air-air interception problem," IEEE Transactions on Aerospace and Electronic Systems, vol. 26, no. 2, pp. 306-325, 1990.

[17] T. Shima, M. Idan, and O. M. Golan, "Sliding-mode control for integrated missile autopilot guidance," Journal of Guidance, Control, and Dynamics, vol. 29, no. 2, pp. 250-260, 2006.

[18] S. R. Kumar, S. Rao, and D. Ghose, "Nonsingular terminal sliding mode guidance with impact angle constraints," Journal of Guidance, Control, and Dynamics, vol. 37, no. 4, pp. 1114-1130, 2014.

[19] C.-K. Ryoo, Y.-H. Kim, and M.-J. Tahk, "Capturability analysis of pn laws using lyapunov stability theory," in AIAA Guidance, Navigation, and Control Conference, Providence, RI, August 2004.
[20] K.-B. Li, H.-S. Shin, and A. Tsourdos, "Capturability of a slidingmode guidance law with finite-time convergence," IEEE Transactions on Aerospace and Electronic Systems, vol. 56, no. 3, pp. 2312-2325, 2019.

[21] M. Guelman, "A qualitative study of proportional navigation," IEEE Transactions on Aerospace and Electronic Systems, vol. 7, no. 4, pp. 637-643, 1971.

[22] A. E. Bryson, "Linear feedback solutions for minimum effort interception, rendezvous, and soft landing," AIAA Journal, vol. 3, no. 8, pp. $1542-1544,1965$

[23] P. Zarchan, Tactical and Strategic Missile Guidance. American Institute of Aeronautics and Astronautics, Inc., 2012.

[24] E. Kreindler, "Optimality of proportional navigation," AIAA Journal, vol. 11 , no. 6, pp. 878-880, 1973.

[25] I.-S. Jeon and J.-I. Lee, "Optimality of proportional navigation based on nonlinear formulation," IEEE Transactions on Aerospace and Electronic Systems, vol. 46, no. 4, pp. 2051-2055, 2010.

[26] T. C. Lee and Z. P. Jiang, "A Generalization of Kasovskii-LaSalle Theorem for Nonlinear Time-Varying Systems: Converse Results and Applications," IEEE Transactions on Automatic Control, vol. 50, no. 8, pp. 1147-1163, 2005.

[27] Khalil, H. K., and J. W. Grizzle, Nonlinear Systems, 3rd ed. Prentice Hall, Upper Saddle River, NJ, 1996.

[28] D. Aeyels and J. Peuteman, "A New Asymptotic Stability Criterion for Nonlinear Time-Variant Differential Equations," IEEE Transactions on Automatic Control, vol. 43, no. 7, pp. 968-971, 1998.

[29] — - "On exponential stability of nonlinear time-varying differential equations," Automatica, vol. 35, no. 6, pp. 1091-1100, 1999.

[30] B.-G. Park, T.-H. Kim, and M.-J. Tahk, "Range-to-go weighted optimal 


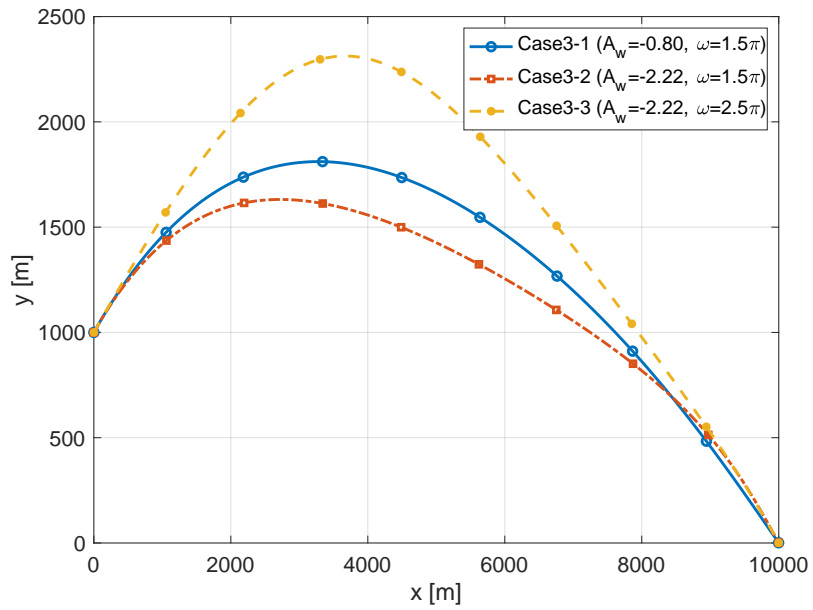

(a) Trajectory
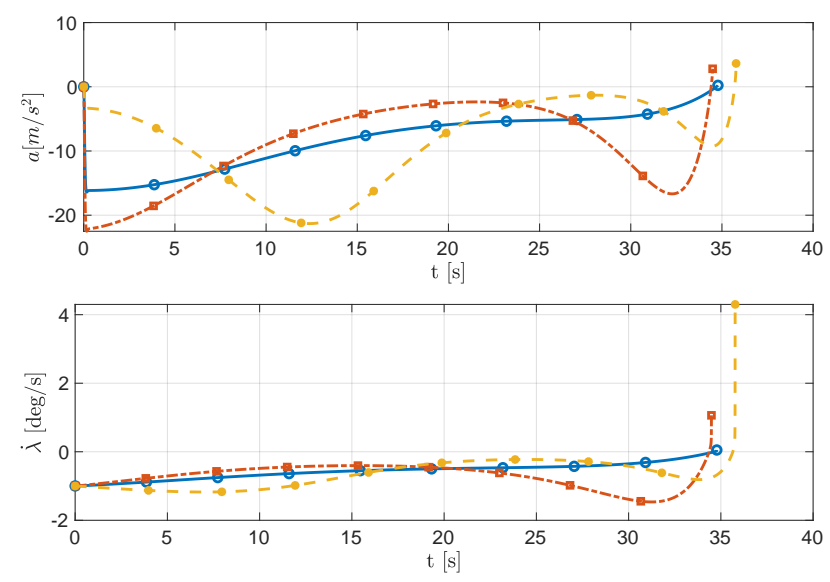

(c) Acceleration and LOS rate
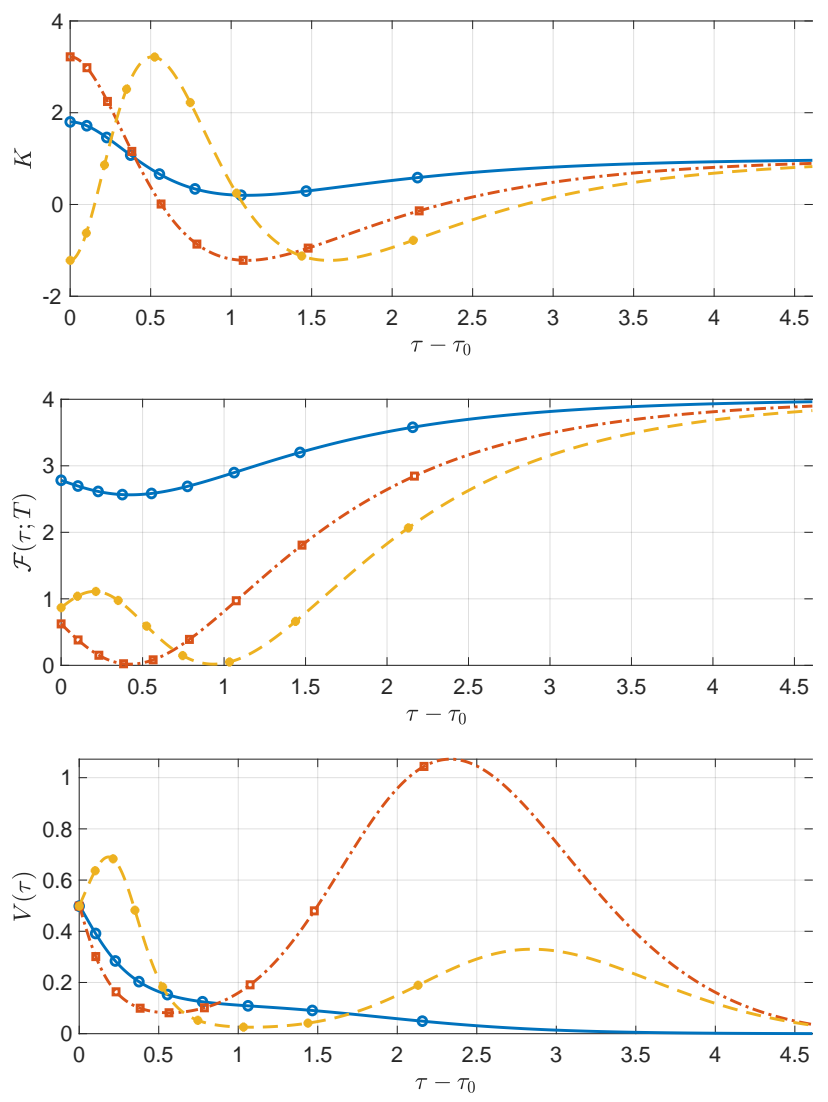

(b) Gain $K, \mathcal{F}(\tau ; T)$, and $V(\tau)$

Fig. 4: Simulation Results for Case 3: Guidance Law with Guaranteed Non-monotonic Convergence

guidance with impact angle constraint and seeker's look angle limits," IEEE Transactions on Aerospace and Electronic Systems, vol. 52, no. 3, pp. 1241-1256, 2016.

[31] I. S. Jeon and J. I. Lee, "Impact-Time-Control Guidance Law With Constraints on Seeker Look Angle," IEEE Transactions on Aerospace and Electronic Systems, vol. 53, no. 5, pp. 2621-2627, 2017.

[32] Y. R. Sharma and A. Ratnoo, "A bearings-only trajectory shaping guidance law with look-angle constraint," IEEE Transactions on Aerospace and Electronic Systems, vol. 55, no. 6, pp. 3303-3315, 2019.

[33] H.-G. Kim and H. J. Kim, "Field-of-view constrained guidance law for a maneuvering target with impact angle control," IEEE Transactions on Aerospace and Electronic Systems, vol. 56, no. 6, pp. 4974-4983, 2020.

[34] D. Mukherjee and S. R. Kumar, "Field-of-view constrained impact time guidance against stationary targets," IEEE Transactions on Aerospace and Electronic Systems, 2021.

[35] T. R. Blackburn, "Method for improving autopilot lag compensation in intercept guidance," Journal of guidance, control, and dynamics, vol. 19, no. 3, pp. 724-726, 1996.

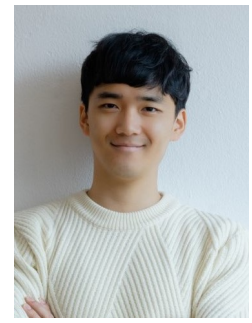

Seokwon Lee received the BSs and PhD degrees in aerospace engineering from the Department of Mechanical and Aerospace Engineering, Seoul National University, Seoul, South Korea, in 2012 and 2019, respectively. He is currently a research fellow in the Centre for Autonomous and Cyber-Physical Systems, School of Aerospace, Transport and Manufacturing, Cranfield University, United Kingdom since November 2020 . He was a postdoctoral researcher with the Institute of Advanced Aerospace Technology, Seoul National University from 2019 to 2020 . His research interests include nonlinear and adaptive control, data-driven control, and guidance and control for flight control systems. 


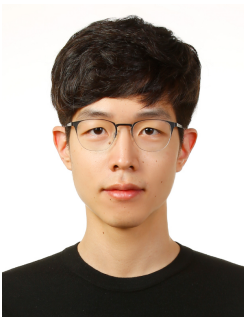

Namhoon Cho is a research fellow in the Centre for Autonomous and Cyber-Physical Systems, School of

Aerospace, Transport and Manufacturing, Cranfield University, United Kingdom, since January 2021. He received his $\mathrm{BSc}$ and $\mathrm{PhD}$ degrees in Mechanical and Aerospace Engineering from Seoul National University, South Korea, in August 2012 and February 2017, respectively. Previously, he was a senior researcher in the 1st R\&D Institute, Agency for Defense Development, South Korea, from March 2019 to December 2020, where he developed guidance and control systems for missiles. He was a research fellow in the Department of Mechanical and Aerospace Engineering, Seoul National University, South Korea, from March 2017 to February 2019. His research interests include robust adaptive control methods based on online model learning, machine learning applications for control and estimation, unified frameworks for automated design of optimal guidance and control systems, and online trajectory optimisation.

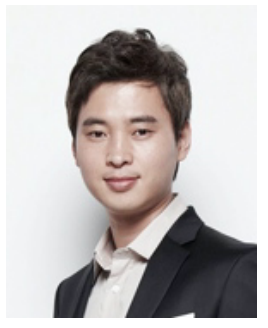

Hyo-Sang Shin received his BSc from Pusan $\mathrm{Na}$ tional University in 2004 and gained an MSc on flight dynamics, guidance and control in Aerospace Engineering from KAIST and a $\mathrm{PhD}$ on cooperative missile guidance from Cranfield University in 2006 and 2010, respectively. He is currently a Professor of Guidance, Control and Navigation (GNC) Systems in Autonomous and Intelligent Systems Group at Cranfield University. His current research interests include target tracking, adaptive and sensor-based of multiple agent systems. control, data-centric GNC, and distributed control 
2021-09-20

\title{
Analysis of guidance laws with non-monotonic line-of-sight rate convergence
}

\author{
Lee, Seokwon
}

IEEE

Lee S, Cho N, Shin H-S. (2022) Analysis of guidance laws with non-monotonic line-of-sight rate convergence. IEEE Transactions on Aerospace and Electronic Systems, Volume 58, Number 2, April 2022, pp. 1029-1041

https://doi.org/10.1109/TAES.2021.3113627

Downloaded from Cranfield Library Services E-Repository 